\title{
LUMBAR SPONDYLOLYSIS IN CHILDREN AND ADOLESCENTS
}

\author{
TETSUKI MORITA, TAKAAKI IKATA, SHINSUKE KATOH, RYOJI MIYAKE
}

From the University of Tokushima, Japan

We investigated 185 adolescents under the age of 19 years with spondylolysis. All but five were active in sport. The pars defect was classified into early, progressive and terminal stages.

Of the 346 pars defects in 185 patients, $39.6 \%$ were early, $29.5 \%$ progressive and $30.9 \%$ in the terminal stages. Conservative management produced healing in 73.0\% of the early, $38.5 \%$ of the progressive and none of the terminal defects.

These results suggest that spondylolysis is caused by repetitive microtrauma during growth and can be successfully treated conservatively if treatment is started in the early stage. There was elongation of the pars interarticularis as the pars defect progressed, and this is likely to be a consequence of the defect rather than a contributing cause.

J Bone Joint Surg [Br] 1995:77-B:620-5.

Received 19 September 1994; Accepted 8 November 1994

Spondylolysis is defined as a defect in the region of the pars interarticularis of the spine. Wiltse, Widell and Jackson (1975) suggested that it represents a 'stress' or fatigue fracture, seen most commonly in children and adolescents. Numerous other reports in the literature support this (Rowe and Roche 1953; Krenz and Troup 1973; Jackson, Wiltse and Cirincione 1976; Taillard 1976; Rosenberg, Bargar and Friedman 1981; Semon and Spengler 1981; O'Neill and Micheli 1989). The theory implies that spondylolysis may be successfully treated conservatively, and several reports have shown that osseous healing of the pars defect can be obtained without surgery (Wiltse et al 1975; Steiner and Micheli 1985; Blanda et al 1993). It is, however, not clear from these reports which treatment is most effective.

T. Morita, MD, Assistant Lecture

T. Ikata, MD, Professor and Director

S. Katoh, MD, Lecturer

R. Miyake, MD, Assistant Lecture

Department of Orthopaedic Surgery, School of Medicine, The University of Tokushima, 3-18-15 Kuramoto-cho, Tokushima 770, Japan.

Correspondence should be sent to Professor T. Ikata.

(01995 British Editorial Society of Bone and Joint Surgery $0301-620 \mathrm{X} / 95 / 41012 \$ 2.00$
We have classified pars defects into three stages and investigated the radiological outcome of conservative treatment using our protocol of bracing. We have also assessed the elongation of the pars interarticularis in relation to the three stages of the defect.

\section{PATIENTS AND METHODS}

We studied the radiological outcome of patients of 18 years of age or younger who presented with lumbar spondylolysis. Between 1988 and 1993, a total of 381 such patients were treated in our clinic; there were 308 boys and 73 girls. The mean age at first presentation was 13.9 years $(6$ to 18$)$. We retrospectively reviewed the progress of pars defects at 193 levels in the 185 patients who had completed our treatment regime.

This large group was collected by screening children with backache who participate in sport. We found one or more pars defects in $32 \%$ of patients under 19 years of age who were referred to our sports clinic with backache.

As far as soccer is concerned, 9832 children under 13 years of age were involved in summer tournaments between 1988 and 1993 from a population of about 800000 and of these 531 had backache with tenderness or pain on extension or a positive straight-leg raising test. From these, 48 of the 220 children seen at our clinic with backache proved to have spondylolysis. Data are not available for other sports.

The mean duration of follow-up was 12.3 months ( 3 to 48). All but five of the 381 patients were very active in sport, but only two gave a definite history of trauma. The sports in which they participated are listed in Table I.

At presentation, the patients were examined and anteroposterior, lateral and oblique radiographs of the lumbosacral spine were taken. We did not use bone scans, believing that CT provides a more accurate diagnosis. We therefore used CT on some patients to confirm defects and to observe the process of healing. Defects of the pars interarticularis were classified into early, progressive and terminal stages (Fig. 1). The early stage was characterised by focal bony absorption or a hair-line defect. In the progressive stage the defect was wide, and small fragments were present. Sclerotic change indicated the terminal stage of development.

All patients were treated conservatively. When spondylolysis was detected, they were advised to discontinue sport and to wear a conventional lumbar corset for three to six months. Follow-up radiographs were taken at four-week 


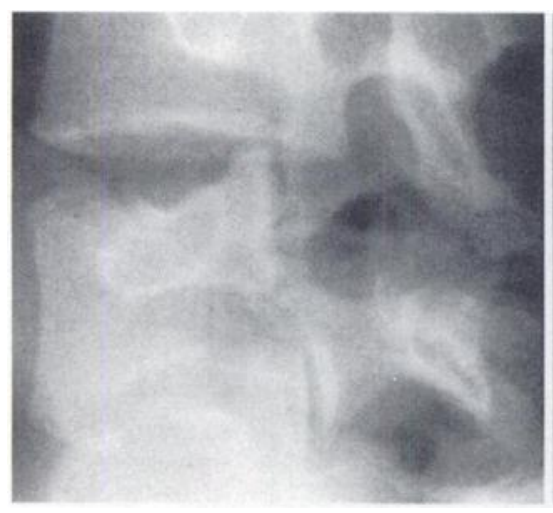

Fig. 1a

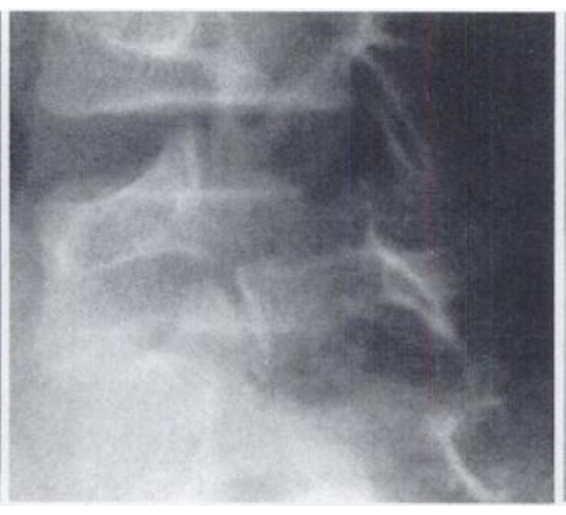

Fig. 1b

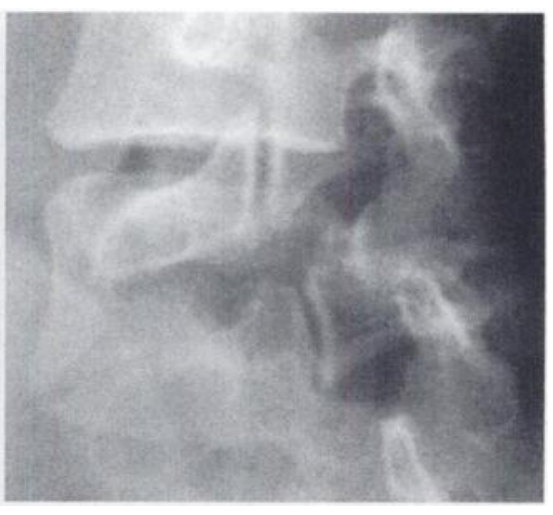

Fig. 1c

Representative radiographs of the early (a), the progressive (b) and the terminal (c) stages of pars defects in oblique views.

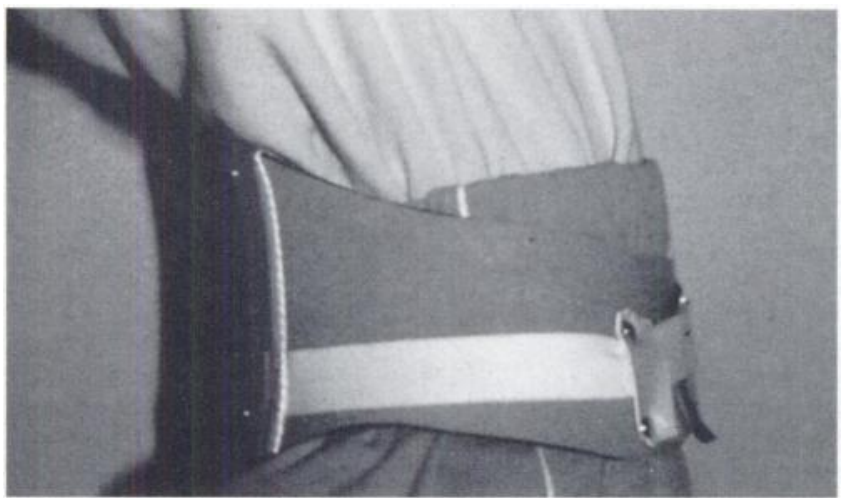

Fig. 2a

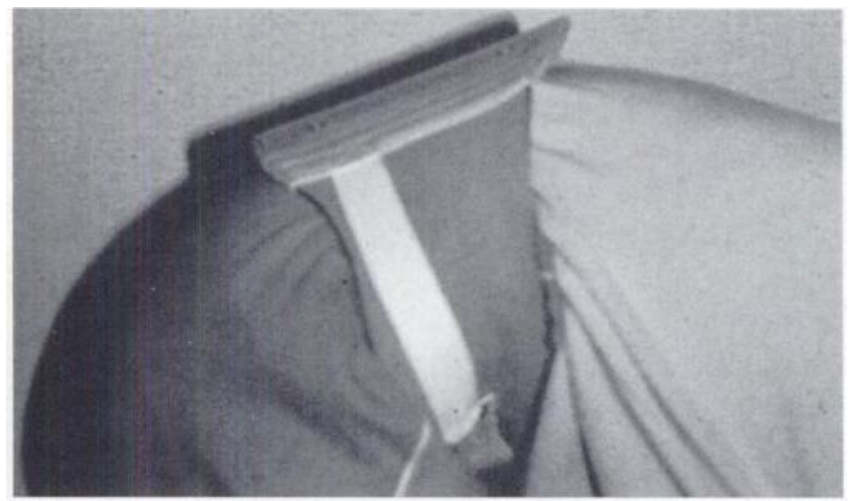

Fig. 2b

Photographs showing the restriction of extension (a) and lack of restriction of flexion (b) produced by a lumbosacral support.

intervals. Once the defects had united, the patients then followed our rehabilitation protocol while wearing a special lumbosacral support. This allows full flexion but provides restriction of extension of the lumbar spine (Fig. 2).

Oblique radiographs and/or CT performed during the course of treatment were used to assess healing of the defects and elongation of the pars interarticularis.

The length of the pars interarticularis at L5 was assessed on a standing oblique view as the distance between a line

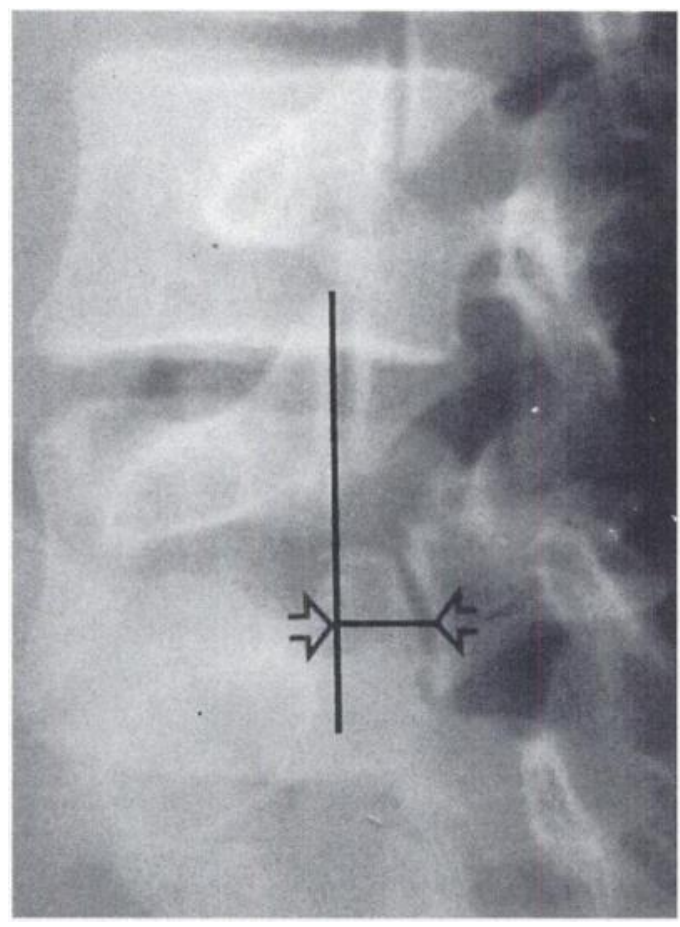

Fig. 3

The length of the pars interarticularis was determined from oblique radiographs as the vertical distance between a line tangential to the joint surface of the superior articular process and the mid-point of the joint surface of the inferior articular process of the same vertebra. To allow for variation we recorded the PD value as the difference in these lengths at L4 and at L5.

tangential to the joint surface of the superior articular process and the mid-point of the joint surface of the inferior articular process of the affected neural arch (Fig. 3). The facet joints were visualised by rotation of the $\mathrm{X}$-ray tube to an angle of between $42^{\circ}$ and $56^{\circ}$.

A preliminary study in normal spines had shown that the coefficient of variation ( $\mathrm{sD} /$ mean) for the length of the pars at L5 was 0.25 , but that the coefficient for the difference between the distances at L4 and L5 was only 0.06 . We 


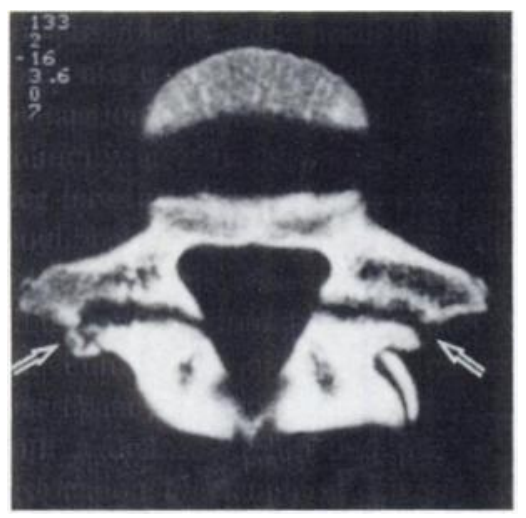

Fig. 4a

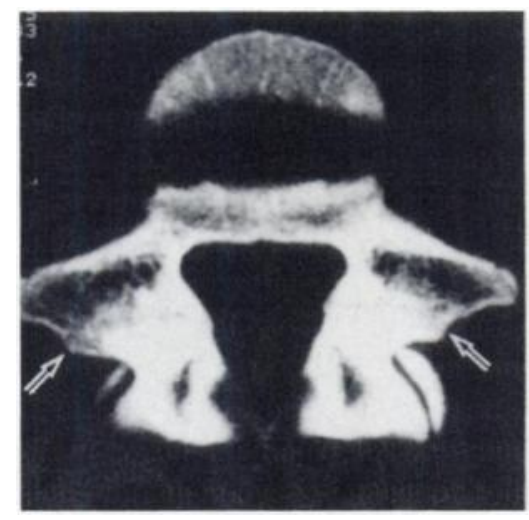

Fig. $4 b$ therefore used this pars difference (PD) in $\mathrm{mm}$ to record our results. The accuracy of these measurements was confirmed by the measurement of 20 radiographs each on two occasions by two independent observers; the results gave interobserver reliability of $\mathrm{p}<0.0001$ (Student's unpaired $t$ test) and intraobserver reliability by coefficient of variation of 0.06. We compared the PD value of the neural arches in patients with pars defects with that in age-matched patients who complained of low back pain but had no pars defects. The angle of lumbar lordosis was measured from the lateral view in the standing position (Wiltse and Winter 1983).

\section{RESULTS}

Of the 185 patients, eight had a pars defect at two levels. Of the 193 defects there was one at $\mathrm{L} 2$, six at $\mathrm{L} 3,16$ at $\mathrm{L} 4,168$ at L5 and two at L6. A unilateral pars defect was found at 40 levels and the remaining 153 levels showed bilateral defects. Of the 346 defects, 137 were in the early stage, 102 were progressive and 107 were in the terminal stage. Bilateral defects were almost always at the same stage. Vertebral slipping of more than 5\% was found in 39 patients at L5, either at presentation or during follow-up. Of these 39 defects, 20 were in the progressive stage and 19 in the terminal stage.

Table I. List of the sports in which the patients were active

\begin{tabular}{ll}
\hline Sports & $\begin{array}{l}\text { Number } \\
\text { of patients }\end{array}$ \\
\hline Soccer & 80 \\
Baseball & 44 \\
Volleyball & 17 \\
Basketball & 11 \\
Athletics & 7 \\
Judo & 5 \\
Others & 16 \\
None & 5 \\
\hline
\end{tabular}

After treatment, bony fusion was diagnosed on the radiographs and/or CT taken at follow-up of three to six months (Fig. 4). Of the 119 defects in the early stage, union was observed in $87(73.0 \%)$. In the progressive stage the incidence of union decreased to 42 of 109 defects (38.5\%). Union failed to occur in all 118 defects in the final stage. These rates of union were significantly different (chisquared test, $\mathrm{p}<0.0001$ ).

At 47 levels with bilateral early-stage defects, bilateral union was achieved in 30 and unilateral union in six. In the progressive stage, bilateral union was achieved at 15 levels of 50 bilateral defects. Unilateral union was seen in 21 of 25 unilateral levels in the early and seven of nine levels in the progressive stage (Table II). The incidence of bilateral union of the defects in the early stage was significantly higher than that in the progressive stage (chi-squared test, $\mathrm{p}<0.01$ ).

The incidence of union of a unilateral defect was also significantly higher than that for bilateral defects (chisquared test, $p<0.05$ ). We found no significant differences in rates of healing of the same-stage defects in the three age groups of under 13,13 to 15 and over 15 years of age.

The mean PD value for the control group was $2.8 \mathrm{~mm}$, which did not differ with age (Fig. 5). The PD value in patients with the early stage of spondylolysis did not differ

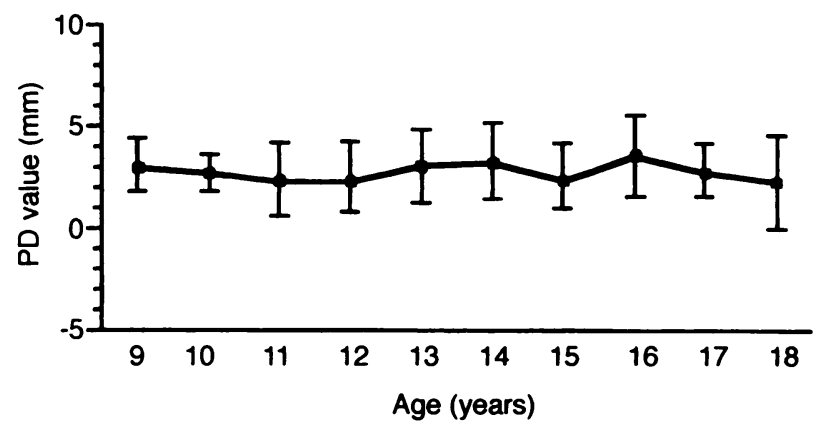

Fig. 5

Relation between the mean PD value ( \pm SD) for each age between 9 and 18 years. 


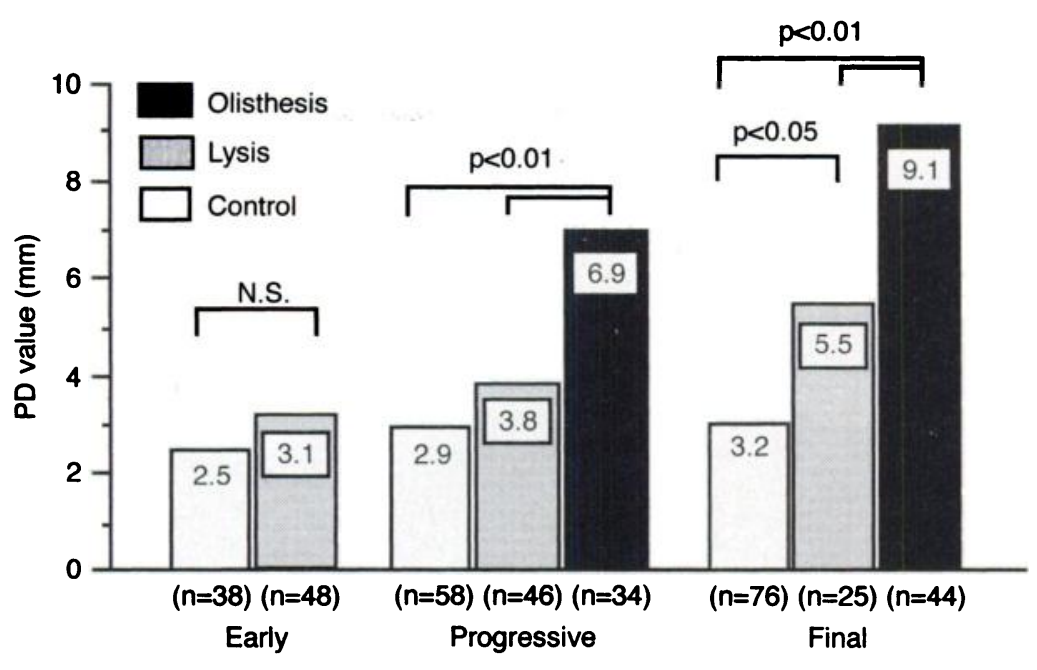

Fig. 6

Relationship between the stages of pars defects and the mean PD values.

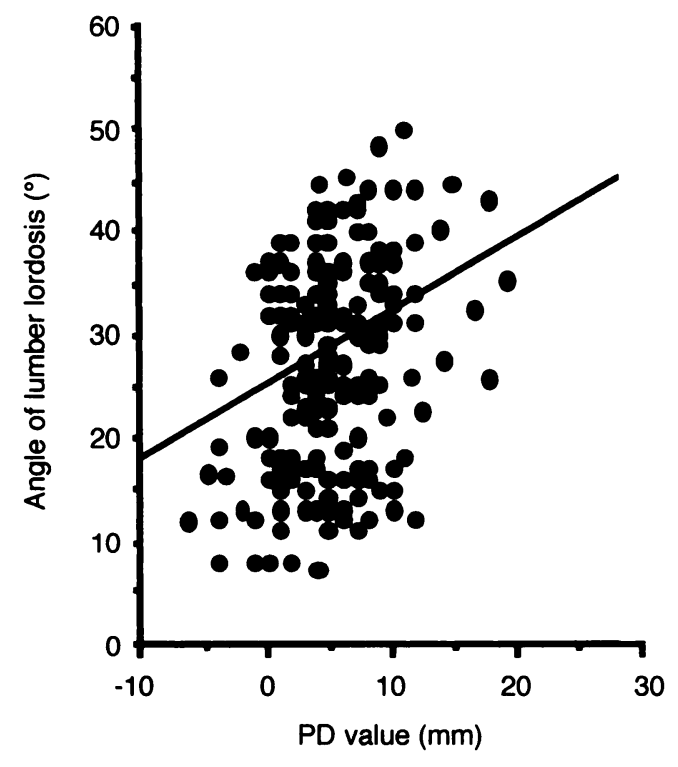

Fig. 7

Scattergraph showing the correlation between the angle of lumbar lordosis and PD value. This was statistically significant using Pearson's correlation coefficient analysis $(p<0.05)$.

Table II. Radiological outcome in patients with pars defects after conservative treatment

\begin{tabular}{lllll}
\hline & & \multicolumn{3}{l}{ Stage of pars defects } \\
\cline { 3 - 5 } Defect & Outcome & Early & Progressive & Final \\
\hline Bilateral & Bilateral union & 30 & 15 & 0 \\
& Unilateral union & 6 & 7 & 0 \\
\multirow{4}{*}{ Unilateral } & Nonunion & 11 & 28 & 56 \\
& Union & 21 & 7 & 0 \\
& Nonunion & 4 & 2 & 6 \\
\hline
\end{tabular}

from that in the control group. In defects at the later stages, the PD value increased even in the absence of vertebral body slip (Fig. 6), but it was obvious that the increase was significant in the presence of olisthesis. It also showed a significant positive correlation with the angle of lumbar lordosis (Fig. 7).

In the 47 patients in whom defects at L5 had united during follow-up, the mean PD value after healing was $2.9 \mathrm{~mm}$ in the cases in the early stage and $4.1 \mathrm{~mm}$ in those in the progressive stage without olisthesis. The latter value was significantly greater than that in the control group (unpaired Student's $t$-test, $\mathrm{p}<0.05$ ). In six patients there was bony union of the defects in a slipped position, and the elongated healed pars in these patients had a mean PD value of $6.8 \mathrm{~mm}$ (Fig. 8).

\section{DISCUSSION}

Hypoplasia or dysplasia of the pars interarticularis has been regarded as the one of the causes of defects in the pars interarticularis (Wiltse, Newman and Macnab 1976; Fredrickson et al 1984), and the relationship between a pars defect and spina bifida has been noted (Eisenstein 1978). Biomechanical analyses have shown that mechanical stress well below the ultimate stress of bone can cause fatigue fracture of the neural arch (Farfan, Osteria and Lamy 1976; Lafferty, Winter and Gambaro 1977; Cyron and Hutton 1978). Clinical evidence has accumulated to support the theory that spondylolysis results from repetitive stresses to the lumbar spine (Rowe and Roche 1953; Krenz and Troup 1973; Jackson et al 1976; Taillard 1976; Rosenberg et al 1981; Semon and Spengler 1981; O'Neill and Micheli 1989). This theory has also been confirmed by evidence of bony healing of the pars defect with non-operative treatment.

Wiltse et al (1975) described 12 of 17 young patients 


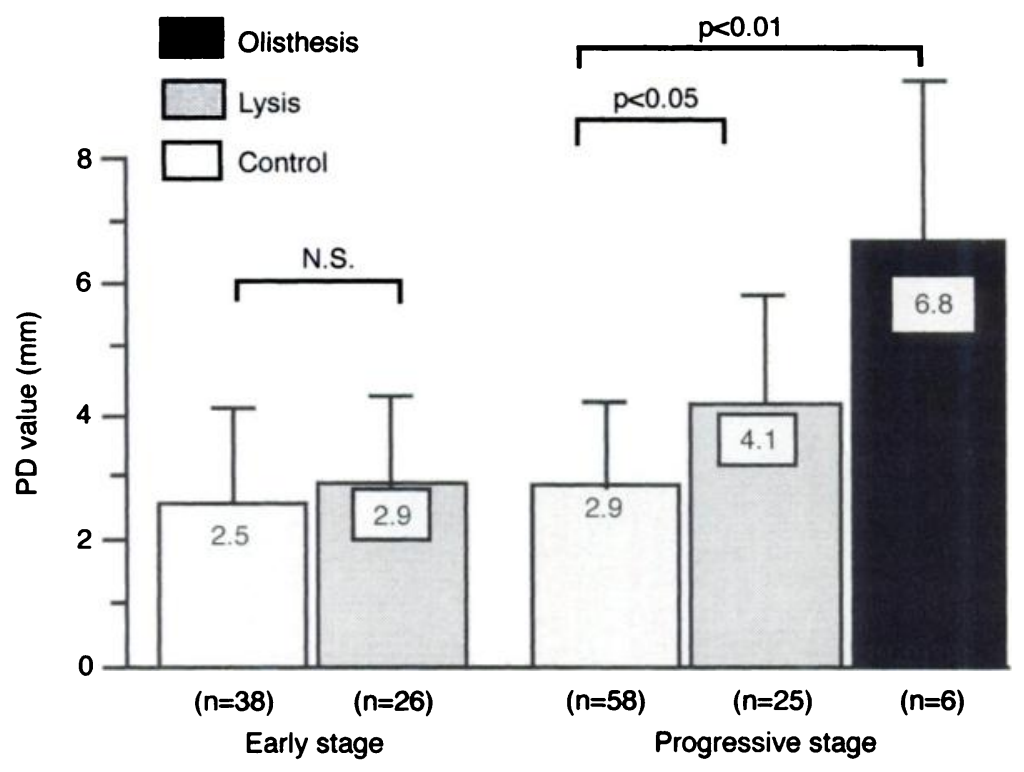

Fig. 8

Relationship between the stages of pars defects and the mean PD values in the cases in which the defects had healed.

who had evidence of healing of pars defects either bilaterally or unilaterally. All but one had been treated by applying casts, braces or corsets. In one patient the defect healed without a brace while continuing gymnastics. Steiner and Micheli (1985) reported radiological evidence of healing of the pars defect in $18 \%$ of 67 patients with symptomatic spondylolysis or grade-one spondylolisthesis treated using a modified Boston brace. Healing occurred in the patients with relatively acute onset of symptoms, but did not correlate with age, delay in treatment or the presence of spina bifida.

In our study, 185 young athletes of 18 years or younger with pars defects were treated by our conservative protocol and studied to estimate healing in relation to the stage of the pars defects. We found that $37.9 \%$ of all pars defects had apparent radiological healing; this is comparable with the outcome of non-surgical treatment reported by Blanda et al (1993). In our series, union was achieved in $73 \%$ of the early-stage defects, $39 \%$ of the progressive-stage defects, and none of the terminal-stage defects. This indicates that pars defects in the immature spine detected at an early stage respond well to conservative treatment without rigid bracing.

Elongation of the pars interarticularis has been considered to result from repeated microfractures and healing of the pars (Jinnaka and Fukumoto 1929; Wiltse 1962). In our series, pars elongation recorded as PD values correlated well with the stages of the defect, the elongation increasing as the defect advanced and again as slipping developed and progressed. This elongation arose before olisthesis was seen, as a result of posterior displacement of the neural arch. This increased later as a consequence of anterior displacement of the vertebral body related to increased lumbar lordosis. We found similar results even in cases in which the defects had healed. The mechanism of the development of vertebral slip in spondylolysis and the influence of a pars defect on the growth of the posterior element of the vertebrae remain to be elucidated (Ikata et al 1995, in press).

Conclusions. Spondylolysis is caused by repetitive microtrauma in the growing child. Lumbar spondylolysis can be successfully treated conservatively if the treatment starts early. Elongation of the pars interarticularis seems to be the consequence of the pars defect rather than the cause of it.

No benefits in any form have been received or will be received from a commercial party related directly or indirectly to the subject of this article.

\section{REFERENCES}

Blanda J, Bethem D, Moats W, Lew M. Defects of pars interarticularis in athletes: a protocol for nonoperative treatment. J Spinal Disord 1993;6:406-11.

Cyron BM, Hutton WC. The fatigue strength of the lumbar neural arch in spondylolysis. J Bone Joint Surg [Br] 1978;60-B:234-8.

Eisenstein S. Spondylolysis: a skeletal investigation of two population groups. J Bone Joint Surg [Br] 1978;60-B:488-94.

Farfan HF, Osteria V, Lamy C. The mechanical etiology of spondylolysis and spondylolisthesis. Clin Orthop 1976;117:40-55.

Fredrickson BE, Baker D, McHolick WJ, Hansen A, Uvbicky JP. The natural history of spondylolysis and spondylolisthesis. J Bone Joint Surg [Am] 1984;66-A:699-707.

Ikata T, Morita T, Katoh S, Miyake R. Pathomechanism of sportsrelated spondylolisthesis in adolescents. Amer J Sports Med 1995; in press.

Jinnaka S, Fukomoto N. Elongation of the neural arch of the fifth lumbar vertebrae. J Jpn Orthop Assoc 1929;145-50.

Jackson DW, Wiltse LL, Cirincione RJ. Spondylolysis in the female gymnast. Clin Orthop 1976;117:68-73. 
Krenz J, Troup JDG. The structure of the pars interarticularis of the lower lumbar vertebrae and its relation to the etiology of spondylolysis: with a report of a healing fracture in the neural arch of a fourth lumbar vertebra. J Bone Joint Surg [Br] 1973;55-B:735-41.

Lafferty JF, Winter WG, Gambaro SA. Fatigue characteristics of posterior elements of vertebrae. J Bone Joint Surg [Am] 1977;59-A: 154-8.

O'Neill DB, Micheli LJ. Postoperative radiographic evidence for fatigue fracture as the etiology in spondylolysis. Spine 1989;14:1342-55.

Rosenberg NJ, Bargar WL, Friedman B. The incidence of spondylolysis and spondylolisthesis in nonambulatory patients. Spine 1981; 6:35-8.

Rowe GG, Roche MB. The etiology of the separate neural arch. $J$ Bone Joint Surg [Am] 1953;35-A:102-10.
Semon RL, Spengler D. Significance of lumbar spondylolysis in college football players. Spine $1981 ; 6: 172-4$.

Steiner ME, Micheli $\mathbf{W}$. Treatment of symptomatic spondylolysis and spondylolisthesis with the modified Boston brace. Spine 1985;10:937-43.

Taillard WF. Etiology of spondylolisthesis. Clin Orthop 1976;117:30-9.

Wiltse LL. The etiology of spondylolisthesis. J Bone Joint Surg [Am] 1962; 44-A:539-60.

Wiltse LL, Newman PH, Macnab I. Classification of spondylolysis and spondylolisthesis. Clin Orthop 1976;117:23-9.

Wiltse LL, Widell EH, Jackson DW. Fatigue fracture: the basic lesion in isthmic spondylolisthesis. J Bone Joint Surg [Am] 1975;57-A:17-22.

Wiltse LL, Winter RB. Terminology and measurement of spondylolisthesis. J Bone Joint Surg [Am] 1983;65-A:768-72. 